\title{
Dedifferentiated endometrial carcinoma: A report of three cases and review of the literature
}

\author{
RYO YOKOMIZO $^{1}$, KYOSUKE YAMADA ${ }^{1}$, YASUSHI IIDA ${ }^{1}$, TAKAKO KIYOKAWA ${ }^{2}$, KAZU UEDA ${ }^{1}$, \\ MOTOAKI SAITO $^{1}$, NOZOMU YANAIHARA ${ }^{1}$, MAYO NAKAMURA ${ }^{2}$ and AIKOU OKAMOTO ${ }^{1}$
}

Departments of ${ }^{1}$ Obstetrics and Gynecology, and ${ }^{2}$ Pathology, The Jikei University School of Medicine, Tokyo 105-8461, Japan

Received May 2, 2017; Accepted August 3, 2017

DOI: $10.3892 / \mathrm{mco} .2017 .1437$

\begin{abstract}
Dedifferentiated endometrial carcinoma, which is defined microscopically as the co-existence of undifferentiated carcinoma and grade 1 or 2 endometrioid adenocarcinoma, is an aggressive type of cancer regardless of the percentage of undifferentiated components. It is reported that undifferentiated carcinoma comprises $9 \%$ of endometrial carcinoma. The percentage of dedifferentiated endometrial carcinoma has been hypothesized to be $40 \%$ of undifferentiated carcinoma. A precise pathological diagnosis is essential for defining the appropriate therapeutic approach and prognosis. Furthermore, since there is an association between dedifferentiated endometrial carcinoma and Lynch syndrome, it is important to identify the patient's genetic background. The current case report presents three cases of dedifferentiated endometrial carcinoma treated in our hospital. In immunohistochemical staining for DNA mismatch-repair (MMR) proteins in dedifferentiated endometrial carcinoma, the components of undifferentiated carcinoma demonstrated a loss of MMR protein expression, and it is suspected that there may be a germline mutation in these cases. Therefore, Lynch syndrome should be suspected and the appropriate genetic approaches in cases of dedifferentiated endometrial carcinoma should be considered.
\end{abstract}

\section{Introduction}

In endometrial carcinoma, undifferentiated carcinoma with grade 1 or 2 endometrioid adenocarcinoma is defined as dedifferentiated endometrial carcinoma (1). Due to a relatively newly recognized entity, there are quite a few cases with dedifferentiated endometrial carcinoma reported worldwide (2).

Correspondence to: Dr Yasushi Iida, Department of Obstetrics and Gynecology, The Jikei University School of Medicine Nishi-Shinbashi 3-25-8, Minato-ku, Tokyo 105-8461, Japan

E-mail: surf-side@hotmail.co.jp

Key words: dedifferentiated endometrial carcinoma, Lynch syndrome, undifferentiated endometrial carcinoma, mismatch-repair, immunohistochemical staining
Of note, there is reportedly an association between dedifferentiated endometrial carcinoma and Lynch syndrome (1), an autosomal dominant inherited cancer susceptibility syndrome caused by MMR genes including MLH1, MSH2, MSH6, and $\operatorname{PMS}(2,3)$.

In the present study, we reported three cases of dedifferentiated endometrial carcinoma treated in our hospital with their immunohistochemical expression of MMR proteins.

\section{Case reports}

Clinical characteristics of three cases. Table I shows the summary of clinical characteristics in three cases of dedifferentiated endometrial carcinoma treated at our hospital in 2014 and 2015. The mean age at diagnosis was 54 years. All three cases presented with atypical genital bleeding as chief complaints and elevated tumor markers (CEA, CA19-9, CA125) were detected. Patients 2 and 3 were null gravid and had familial histories of colon cancer. As for past medical history, patient 1 had a history of ulcerative colitis and patient 3 had a history of renal cell carcinoma. Preoperative endometrial biopsies were performed in all the patients and histological type was endometrioid adenocarcinoma G1 in patient 1 and high-grade adenocarcinoma in patient 3 . In patient 2, we did not pick up sufficient materials. All three patients underwent surgery based on the diagnosis of endometrial carcinoma. In patients 1 and 3 , we accomplished complete surgery without any residual tumor. By contrast, we did not accomplish complete surgery in patient 2 as there were many unresectable tumors in the retroperitoneal cavity. Patient 1 was early stage, and patients 2 and 3 were advanced stage. The treatment strategy for adjuvant therapy was different in the patients because of different degrees of renal dysfunction: It was mild in patient 1 , moderate in patient 2 , and severe in patient 3. Patient 1 was alive with no evidence of disease 2 years post-operation, but patients 2 and 3 succumbed to the disease at 5 months and 7 months post-operation, respectively.

The patients provided permission to publish these features of her case, and the identity of the patient has been protected. Furthermore, ethics approval was obtained from the Ethics Comittee of the Jikei University School of Medicine [approval no. 14-132(4001)] and written informed consent was obtained from the patient for publication of this case study and the accompanying images. 
Table I. Patient characteristics.

\begin{tabular}{|c|c|c|c|}
\hline & Patient 1 & Patient 2 & Patient 3 \\
\hline Age at diagnosis (years) & 66 & 48 & 48 \\
\hline Pregnancy history & $3 \mathrm{G} 2 \mathrm{P}$ & 0G0P & 0G0P \\
\hline Family history & None & Father: Colon cancer & Father: Colon cancer \\
\hline Past history & Ulcerative colitis & None & Renal cell carcinoma \\
\hline Chief complaint & Atypical genital bleeding & Atypical genital bleeding & Atypical genital bleeding \\
\hline $\begin{array}{l}\text { Preoperative endometrial } \\
\text { biopsy }\end{array}$ & $\begin{array}{l}\text { Endometrioid } \\
\text { adenocarcinoma G1 }\end{array}$ & Insufficient material $^{\mathrm{a}}$ & $\begin{array}{l}\text { High-grade } \\
\text { adenocarcinoma }\end{array}$ \\
\hline Operation & $\begin{array}{l}\mathrm{TAH}+\mathrm{BSO}+ \\
\mathrm{LNX} \text { (pelvis-paraaorta) }\end{array}$ & $\begin{array}{l}\text { TAH + BSO + OMTX + } \\
\text { Right hemicolectomy + } \\
\text { Hartmann operation }\end{array}$ & $\begin{array}{l}\text { TAH + BSO + OMTX + } \\
\text { LNX (pelvis) + } \\
\text { LNS (paraaorta) }\end{array}$ \\
\hline FIGO stage & IA & IVB & IIIA \\
\hline TNM classification & pT1aN0M0 & pT4bNXM0 & pT3aNOM0 \\
\hline \multirow[t]{2}{*}{$\begin{array}{l}\text { Carcinoma components } \\
\text { confirmed in } \\
\text { hysterectomy specimen }\end{array}$} & Endometrioid G1: 55\% & Endometrioid G1: 10\% & Endometrioid G1: 40\% \\
\hline & Undifferentiated: $45 \%$ & Undifferentiated: $90 \%$ & Undifferentiated: $60 \%$ \\
\hline Residual tumor & None & $>5 \mathrm{~cm}$ above ureter & None \\
\hline \multirow[t]{3}{*}{ Adjuvant therapy } & AP protocol & TC protocol & Radiation \\
\hline & $\begin{array}{l}\text { Adriamycin: } 60 \mathrm{mg} / \mathrm{m}^{2} \text {, } \\
\text { Cisplatin: } 50 \mathrm{mg} / \mathrm{m}^{2}\end{array}$ & $\begin{array}{l}\text { Paclitaxel: } 180 \mathrm{mg} / \mathrm{m}^{2} \text {, } \\
\text { Carboplatin: AUC } 6\end{array}$ & Pelvis \\
\hline & every 3 weeks, 6 cycles & every 4 weeks, 4 cycles & $50 \mathrm{~Gy}$ \\
\hline Progression-free time & 2 years & 5 months & 5 months \\
\hline $\begin{array}{l}\text { Recurrent or } \\
\text { metastases sites }\end{array}$ & None & $\begin{array}{l}\text { Enlargement of pelvic } \\
\text { tumor }\end{array}$ & Lung, vaginal stump \\
\hline Outcome & Alive & $\begin{array}{l}\text { Death } 5 \text { months after } \\
\text { operation }\end{array}$ & $\begin{array}{l}\text { Death } 7 \text { months after } \\
\text { operation }\end{array}$ \\
\hline
\end{tabular}

TAH, total abdominal hysterectomy; BSO, bilateral salpingo-oophorectomy; LNX, lymphadenectomy; OMTX, omentectomy; LNS, lymph node sampling. ${ }^{a}$ Impression of preoperative MRI imaging was uterine serous carcinoma or carcinosarcoma.

Table II. Immunohistochemical analyses of dedifferentiated endometrial carcinoma cases.

\begin{tabular}{lccc}
\hline & MLH1 & MSH2 & MSH6 \\
\hline Case 1 (DC) & Negative & Strongly positive & Strongly positive \\
Case 2 (DC) & Negative & Strongly positive & Strongly positive \\
Case 3 (DC) & Negative & EM: Negative & EM: Weakly positive \\
& & UC: Strongly positive & UC: Strongly positive \\
Case 4 (Serous) & Strongly positive & Strongly positive & Strongly positive \\
control case) & & & Strongly \\
\end{tabular}

DC, dedifferentiated carcinoma; UC, undifferentiated carcinoma; EM, endometrioid adenocarcinoma.

Pathological findings. The histological examination performed in all three cases revealed endometrial carcinoma containing low-grade endometrioid adenocarcinoma and undifferentiated carcinoma, with the abrupt transition of any two components showing a sharp border (Fig. 1). The amount of undifferentiated carcinoma components varied among the cases, ranging from 45 to $90 \%$ (Table I). Immunohistochemically, the expression for ER, PR, and p53 was similar in all three cases of dedifferentiated carcinoma: ER and PR were positive in the endometrioid adenocarcinoma component, and negative for the undifferentiated carcinoma component, while p53 was overexpressed only in the undifferentiated carcinoma component (Fig. 1).

We also performed immunohistochemistry for four DNA MMR proteins, i.e., MLH1, MSH2, MSH6, and PMS2, which served as surrogate markers for Lynch syndrome, in three 

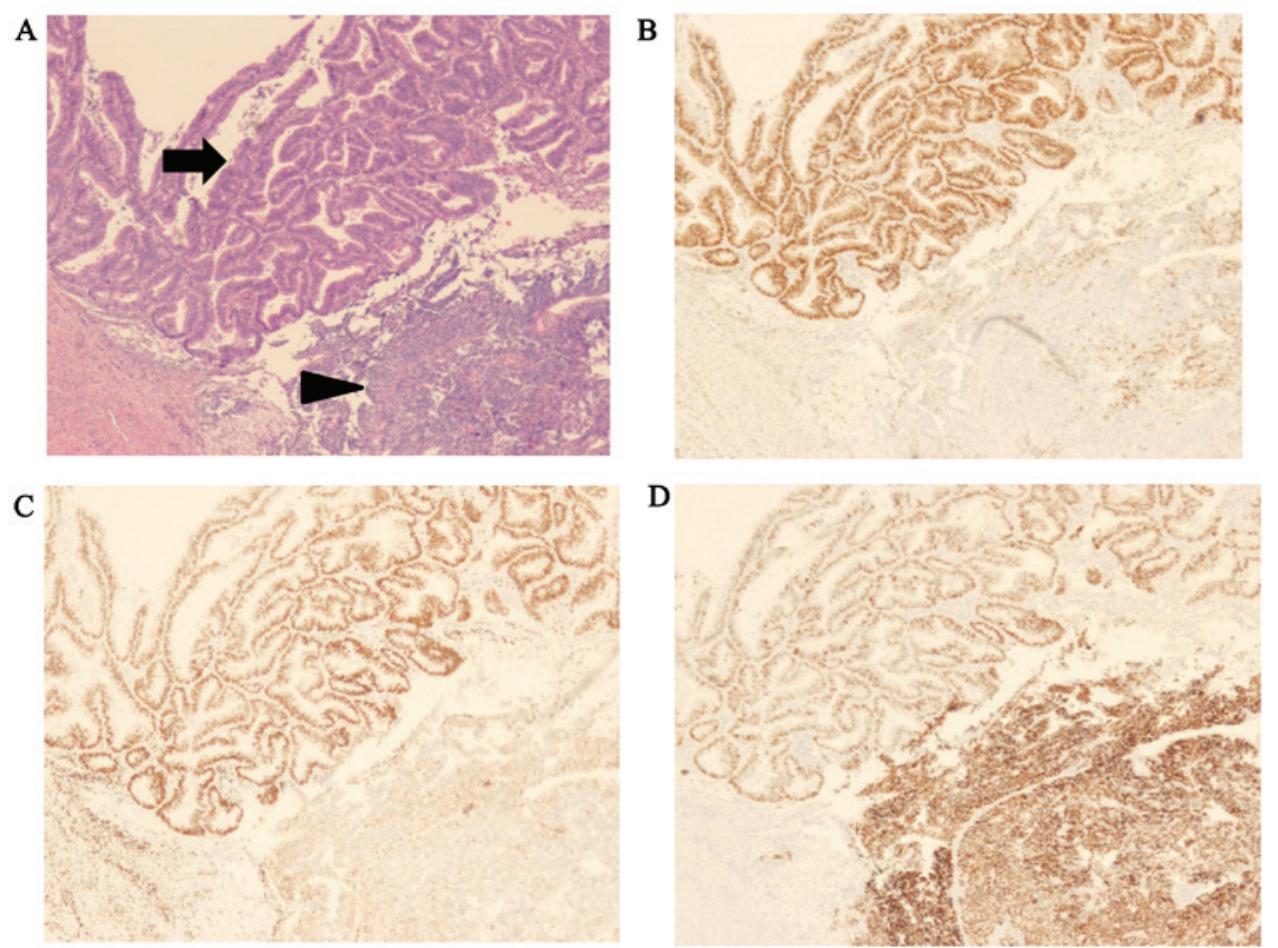

Figure 1. Pathological findings of dedifferentiated endometrial carcinoma. The left upper area (arrow) is composed of fused glandular component and is thought to be endometrioid adenocarcinoma grade 1. On the other hand, the right lower area (arrowhead) shows cells with high nuclear/cytoplasmic (N/C) ratio proliferating without any differentiation and is thought to be undifferentiated carcinoma. According to these findings, this endometrial carcinoma is classified as dedifferentiated endometrial carcinoma (A). For immunohistochemistry, endometrioid adenocarcinoma shows ER (+) and PR (+), and p53 (-) (left upper area). Undifferentiated carcinoma shows ER (-) and PR (-), and p53 (++) (right lower area) (B, C, D). Original magnification, x40.

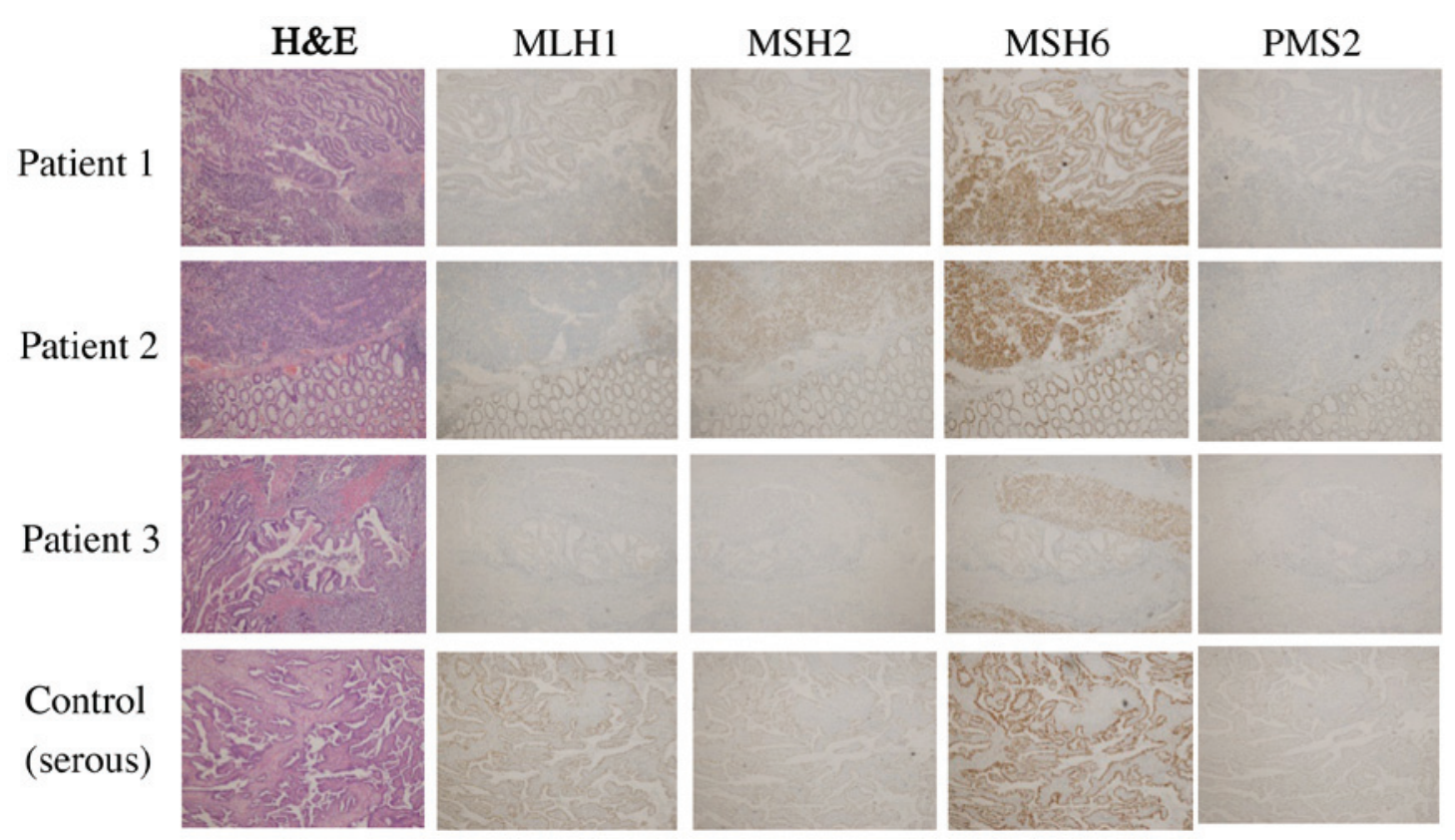

Figure 2. Immunohistochemical analyses of the cases of dedifferentiated endometrial carcinoma. Images show immunohistochemical analyses of the cases and a control (serous). The assessments are summarized in Table II (4). Original magnification, $\mathrm{x} 40$.

cases of dedifferentiated carcinoma described above and the case of serous carcinoma (control) (Fig. 2 and Table II) (4). The undifferentiated carcinoma component in three cases of dedifferentiated carcinoma showed loss of MLH1/PMS2. These four DNA MMR proteins were retained in all the serous carcinoma cases.

\section{Discussion}

In 2006, Silva et al reported cases of endometrial carcinoma in which low-grade endometrioid carcinoma was combined with undifferentiated carcinoma, and designated them as dedifferentiated endometrial carcinoma (5). The rate of each 
component was not defined. It is reported that undifferentiated carcinoma comprises $9 \%$ of endometrial carcinoma (5). The percentage of dedifferentiated endometrial carcinoma is thought to be $40 \%$ of undifferentiated carcinoma (5). The peak age of dedifferentiated endometrial carcinoma is 55 years, and the primary complaint is post-menopausal atypical genital bleeding (1). The risk factor remains unclear but some case reports have shown an association with Lynch syndrome (1). According to Silva's report, the frequency of stage I and II was $37.5 \%$ and stage III and IV was $62.5 \%$ (5). The clinical characteristics of our cases are similar to previous reports.

The pathological characteristics of undifferentiated carcinoma are as follows: Proliferation of small- to middle-size cells without any differentiation; typically tumor cells are positive for p53, EMA, CK18, and vimentin, negative for ER, PR, or E-cadherin, and they may be negative for pan-cytokeratins (1). Undifferentiated carcinoma may arise through transformation or dedifferentiation in well-differentiated endometrioid adenocarcinoma (5). According to the study by $\mathrm{Wu}$ et $\mathrm{al}$, when dedifferentiated endometrial carcinoma metastasizes, the majority of metastases are comprised of the undifferentiated component. In the metastatic lesions, ER and PR expression may be the tissue biomarkers to distinguish the origin of the tumor (6). Hoang et al also reported that the loss of PAX8 and ER expression may be a fundamental feature of dedifferentiation (7). There is a tendency for the well-differentiated endometrioid component to exist mainly on the tumor surface and for the undifferentiated component to exist in the deeper area (8). Due to this localization, it is possible that the undifferentiated component cannot be identified by biopsy; thus, an exact diagnosis and the appropriate operation are difficult to determine. In the current report, there were no cases of exact diagnosis using a biopsy specimen. According to Kanis et al, the sensitivity of the preoperative endometrial biopsy or curettage decreases with high-risk histology endometrial cancer (9). It also has been demonstrated that undifferentiated carcinoma component when coexisting with endometrioid adenocarcinoma may be erroneously recognized as solid component of endometrioid adenocarcinoma, leading to misdiagnose the tumor as FIGO grade 2 or 3 endometrioid adenocarcinoma (2). While the tumors cells are discohesive with high-grade nuclear feature and grow in a sheet-like manner in undifferentiated carcinoma, those of endometrioid adenocarcinoma forming solid nests are cohesive and show similar cytology to those forming glands. Previous findings suggest the strategy to distinguish between undifferentiated carcinoma and solid component of endometrioid adenocarcinoma. When an undifferentiated carcinoma component is juxtaposed with low-grade endometrioid adenocarcinoma, a sharp boundary is evident between them, whereas a seamless transition from glandular component to solid component is observed in high-grade endometrioid adenocarcinoma (10). Ramalingan et al reported that PAX8 may be an effective biomarker to distinguish undifferentiated carcinoma (11).

The endometrioid component was ER (+) and PR (+), and p53 (-). The undifferentiated component was ER (-) and PR (-), and p53 (++) (Fig. 1). These findings are characteristic of type 1 and type 2 cancer coexistence (12). Furthermore, all the components of undifferentiated carcinoma in dedifferenti- ated carcinoma showed loss of MLH1/PMS2, whereas serous adenocarcinoma was positive. Dedifferentiated carcinoma has been reported to be associated with Lynch syndrome (1). Lynch syndrome is an autosomal dominant inherited cancer susceptibility syndrome caused by germline mutations in one of a set of MMR genes (MLH1, MSH2, MSH6, and PMS (2,3). Loss of expression is a predictive marker for germline mutation. MLH1 dimerizes with PMS2 in functional states, in order that MLH1 abnormality is accompanied by the loss of PMS2. Garg et al reported that five of seven dedifferentiated carcinomas were associated with abnormalities in MLH1/PMS2 (13). However, loss of MLH1 is caused by methylation of MLH1 as well as germline mutations of MLH1. They did not perform genetic testing for cases with abnormalities in MLH1/PMS2. In the study by Lu et al on endometrial cancer at age younger than 50 years, only one of 13 cases with loss of MLH1 had germline mutation of MLH1 and the other cases had methylation of MLH1 (14). Personal and family history is very important for identifying patients with high risk of Lynch syndrome (3). In the same study, they also reported that women with a Lynch syndrome-associated cancer had a $43 \%$ chance of germline mutation in MMR as compared to women without an affected first-degree relative (14). Two of our three cases having family history of colon cancer in a first-degree relative, were referred for genetic counseling. According to the Berretta et al, most of the patients diagnosed with dedifferentiated endometrial carcinoma were deceased due to disease within one year, and the appropriate treatment for dedifferentiated endometrial carcinoma was not defined (15). In most reports, operative therapy with adjuvant chemotherapy was performed, but there is no evidence-based strategy, including operative therapy, chemotherapy, and radiation therapy (15). In general, the prognosis of dedifferentiated endometrial carcinoma is poor regardless of the undifferentiated component percentage and the degree of differentiation of endometrioid adenocarcinoma (4). The concept of the rare histological type should be recognized when seeking a precise prognostic analysis and the appropriate therapeutic strategy. In addition, personal and family history and immunohistochemical analysis of MMR protein for patients with dedifferentiated carcinoma of endometrium should be considered to identify the risk of Lynch syndrome.

\section{Acknowledgements}

The present study has undergone English language review by a native English speaker (Enago).

\section{References}

1. Kurman RJ, Carcangiu ML, Herrington CS, Young and RH: WHO classification of tumours of female reproductive organs. Fourth Edition. World Health Organization: 132-133, 2014.

2. Shen Y, Wang Y, Shi Y, Liu J and Liu Y: Clinicopathologic study of endometrial dedifferentiated endometrioid adenocarcinoma: A case report. Int J Clin Exp Pathol 5: 77-82, 2012.

3. Lynch HT and de la Chapelle A: Genetic susceptibility to non-polyposis colorectal cancer. J Med Genet 36: 801-818, 1999.

4. Kazu U, Kyosuke Y, Mitsuyoshi U, Yoshio I, Misako S, Takashi N, Hiroyuki T, Aikou O, Misato S, et al: Association of extracellular matrix metalloproteinase inducer in endometrial carcinoma with patient outcomes and clinicopathogenesis using monoclonal antibody 12C3. Oncol Rep 17: 731-735, 2006. 
5. Silva EG, Deavers MT, Bodurka DC and Malpica A: Association of low-grade endometrioid carcinoma of the uterus and ovary with undifferentiated carcinoma: A new type of dedifferentiated carcinoma? Int J Gynecol Pathol 25: 52-58, 2006.

6. Wu ES and Shih Le-M and Díaz-Montes TP: Dedifferentiated endometrioid adenocarcinoma: An under-recognized but aggressive tumor?. Gynecol Oncol Rep 5: 25-27, 2013.

7. Hoang LN, Lee YS, Karnezis AN, Tessier-Cloutier B, Almandani N, Coatham M, Gilks CB, Soslow RA, Stewart CJ, Köbel $\mathrm{M}$ and Lee CH: Immunophenotypic features of dedifferentiated endometrial carcinoma - insights from BRG1/INI1-deficient tumours. Histopathology 69:560-569, 2016.

8. Tafe LJ, Garg K, Chew I, Tornos C and Soslow RA: Endometrial and ovarian carcinomas with undifferentiated components: Clinically aggressive and frequently underrecognized neoplasms. Mod Pathol 23: 781-789, 2010.

9. Kanis MJ, Rahaman J, Moshier EL, Zakashansky K, Chuang L and Kolev V: Detection and correlation of pre-operative, frozen section, and final pathology in high-risk endometrial cancer. Eur J Gynaecol Oncol 37: 338-341, 2016.

10. Jiheun H, Eun YK, Sung ER, Soo YH and Ahwon L: Dedifferentiated endometrioid carcinoma of the uterus : report of four cases and review of literature. World J Surg Oncol 15: 17, 2017.

11. Ramalingam P, Masand RP, Euscher ED, Malpica A: Undifferentiated carcinoma of the endometrium: an expanded immunohistochemical analysis including PAX-8 and basal-like carcinoma surrogate markers 35. Int J Gynecol Pathol: 410-418, 2016.
12. Bokhman JV: Two pathogenetic types of endometrial carcinoma. Gynecol Oncol 15: 10-17, 1983.

13. Garg K, Leitao MM Jr, Kauff ND, Hansen J, Kosarin K, Shia J and Soslow RA: Selection of endometrial carcinomas for DNA mismatch repair protein immunohistochemistry using patient age and tumor morphology enhances detection of mismatch repair abnormalities. Am J Surg Pathol 33: 925-933, 2009.

14. Lu KH, Schorge JO, Rodabaugh KJ, Daniels MS, Sun CC, Soliman PT, White KG, Luthra R, Gershenson DM and Broaddus RR: Prospective determination of prevalence of lynch syndrome in young women with endometrial cancer. J Clin Oncol 25: 5158-5164, 2007.

15. Berretta R, Patrelli TS, Faioli R, Mautone D, Gizzo S, Mezzogiorno A, Giordano G and Modena AB: Dedifferentiated endometrial cancer: An atypical case diagnosed from cerebellar and adrenal metastasis: Case presentation and review of literature. Int J Clin Exp Pathol 6: 1652-1657, 2013. 